\title{
Studies of Conductive and Dielectrical Properties of Polymeric Charge-Transfer Complexes. I. Polycation-TCNQ Salts
}

\author{
Shinobu Ikeno, ${ }^{*}$ Keiichi Matsumoto, ${ }^{* *}$ Masaaki Yokoyama, ${ }^{* *}$ \\ and Hiroshi Mikawa** \\ *On leave of absence from Research Division, Matsushita Electric \\ Works Co. Ltd., Kadoma, Osaka 571, Japan. \\ **Department of Applied Chemistry, Faculty of Engineering, \\ Osaka University, Suita, Osaka 565, Japan.
}

(Received October 8, 1976)

\begin{abstract}
Conductive and dielectrical properties of TCNQ anion radical salt (II) and complex salts (III) of<smiles>CC(C)(C)Cc1ccc(C[N+](C)(C)C[N+](C)(C)C(C)(C)C)cc1</smiles>

were studied. Conductivity and dielectric constant were larger in (III). The dielectric constants of (III) were $70-7000$ at $30 \mathrm{kHz}-1 \mathrm{MHz}$ at room temperature; they increased with temperature and decreased with increasing frequency and $\mathrm{AC}$ field strength. The activation energies of polarization agreed very closely with those of electrical conduction, suggesting the same mechanistic origin for both phenomena. To explain these results, a model has been proposed for the structure of polycation-TCNQ salts-in which the TCNQ molecules are packed randomly in the polycation network and every TCNQ molecule has the possibility of making electrical contact with nearby TCNQ molecules, depending on the degree of the volume content of TCNQ in the material. Eventually, long sequences of TCNQ molecules connecting the two electrodes can arise in this model and these sequences provide electrical conductivity of the materials. Also, isolated shorter sequences are possible in the matrix, and these contribute to the polarization. Thus, the dielectric polarization of the material can be explained by a microscale Maxwell-Wagner polarization mechanism.

KEY WORDS Semiconducting Polymers / Dielectric Constant / Maxwell-Wagner Polarization / Polycation-TCNQ Salt / Simple Salt / Complex Salt / Doping / Polarization / Column / Gelation Theory /
\end{abstract}

While there have been a significant number of studies of semiconducting polymers, ${ }^{1,2}$ little attention has been paid to their dielectrical properties.

Pohl, et al., ${ }^{3,4,5}$ studied the dielectrical properties of semiconducting polyacene radical quinon polymers, and found that these materials exhibited extraordinary high dielectric constants. To explain this property, they proposed a "hyperelectronic" polarization, which may in principle be looked upon as a Maxwell-Wagner polarization of molecular scale. Such a polarization is usually expected in microscopically heterogeneous dielectrics, i.e., suspensions of conducting particles in which the charge carriers can move under the influence of an external field.

There are many reports on the conductive properties of the highly conductive salts of tetracyanoquinodimethane (TCNQ) anion radical with different kinds of monomeric ${ }^{6}$ and polymeric ${ }^{7}$ cations. In the field of polymer materials, 
Mizoguchi, ${ }^{8}$ et al., have investigated TCNQ salts of polycations having cationic sites in the main chain and have proposed a conduction mechanism in such materials. Recently, Tsubomura, et al. ${ }^{9}$ proposed another conduction mechanism in poly(4-vinylpyridinium)-TCNQ salts, based on the electronic absorption spectra. However, there are few reports on the dielectrical properties of such polymeric TCNQ salts. Investigation of this property will, therefore, be worthwhile and the elucidation of the polarization mechanism in connection with the conductiom mechanism will be very interesting.

In the present study, we report that $\operatorname{poly}(N$, $N, N^{\prime}, N^{\prime}$-tetramethylhexamethyleneparaxylene diammonium)-TCNQ salts exhibit high dielectric constants and that the electronic carriers are responsible for both electrical conduction and dielectric polarization. In order to explain the observed electrical properties, a model has been presented for the structure of polycation-TCNQ salts and this model has been treated statistically by applying the method developed by Flory for explaining the gelation of polymers in solution.

\section{EXPERIMENTAL}

\section{Synthesis}

$\operatorname{Poly}\left(N, N, N^{\prime}, N^{\prime}\right.$-tetramethylhexamethylene- paraxylene diammonium)-TCNQ salt (simple salt) (II) and the complex salts (III) which include additional doped neutral TCNQ were synthesized according to the method in the literature. ${ }^{10}$ The synthetic scheme is illustrated in Figure 1.

\section{Measurements}

Although the obtained simple salt and complex salt were film-forming, they were too brittle to make good film specimens for dielectric measurements. Therefore, finely ground polymers pressed into small pellets at $300 \mathrm{~kg} / \mathrm{cm}^{2}$ for $30 \mathrm{~min}$ in vacuum were used for the measurements. The thickness of the pellets was about $1.0 \mathrm{~mm}$. Gold was evaporated onto the surfaces of the pellets to form two terminal electrodes.

Measurements of the parallel capacitance and resistance were made at frequencies ranging from $30 \mathrm{~Hz}$ to $1 \mathrm{MHz}$ at temperatures ranging from $-196^{\circ} \mathrm{C}$ to $60^{\circ} \mathrm{C}$, with a mutual conductance bridge (Ando Electric Co., Model TR-1C). Owing to the high dielectric constant and conductivity of the polymers, we used external capacitors and resistors (Ando Electric Co., YS-1 GC Box) to extend the measuring ranges up to $11 \mathrm{nF}$ and $11000 \mu \mho$, respectively. Since we omitted the guard electrode, dielectric constant and loss tangent values were corrected according to the ASTM Standard.*

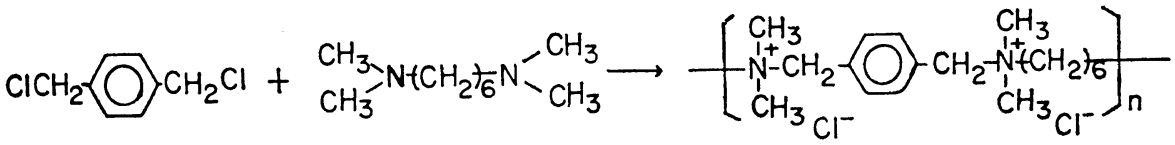

$$
\begin{aligned}
& \text { Polycation Chloride [I] }
\end{aligned}
$$

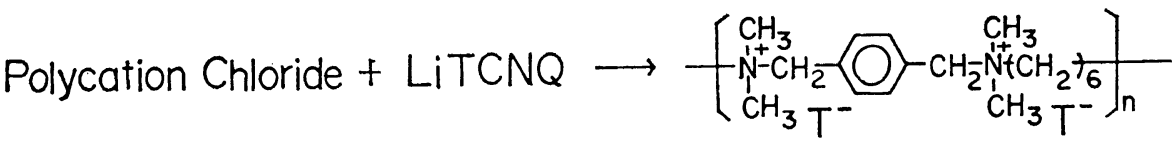

$$
\begin{aligned}
& \text { Simple Salt [II] }
\end{aligned}
$$

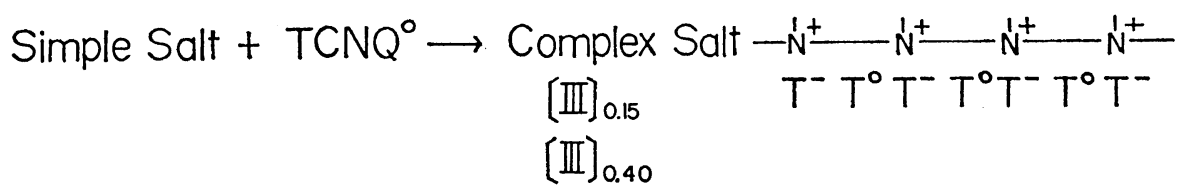

Figure 1. Schematic representation of polymer synthesis.

* ASTM Standard Part 29 "Electrical Insulating Materials" p 78 (1967). 
Electrical conductivity was measured with a Takeda-Riken Industry Model TR-8651 Electrometer.

\section{RESULTS AND DISCUSSION}

\section{Conductive and Dielectric Properties}

The specific resistivities and activation energies for the electrical conduction of sample II (simple salt) and two complex salts, sample III $_{0.15}$ (doping ratio, ${ }^{*} 0.15$ ) and sample III $_{0.40}$ (doping ratio, 0.40), are shown in Table I and Figure 2. Upon increasing the neutral TCNQ, the room temperature specific resistivity decreases from $2.5 \times 10^{7}$ to $6.0 \times 10^{3} \Omega \mathrm{cm}$, which are approximately consistent with the results reported by Mizoguchi. ${ }^{8}$

While a plot of the logarithm of the resistivity against $1 / T$ is linear, breaks were found around $-85^{\circ} \mathrm{C}$, with lower activation energies in the low temperature region. Although such breaks are often observed in semiconducting polymers, ${ }^{2}$ and the impurity effect including oxygen or the glass transition phenomena have been considered in some cases to be the reason for such breaks, the explanation is not yet clear.

The relative dielectric constants, $\varepsilon_{\mathrm{r}}$ of samples I, II, $\mathrm{III}_{0.15}$, and $\mathrm{III}_{0.40} v s$. frequency at various temperatures are shown in Figures 3-5. As in the case of the electrical conductivity, the dielectric constant increases with increasing amount of the doped neutral TCNQ. In comparison with the low dielectric constants, mostly between 2 and 10, for ordinary polymers, the observed dielectric constants are fairly large. As is seen in the figures, the dielectric constant increases with temperature and decreases in the high frequency region.

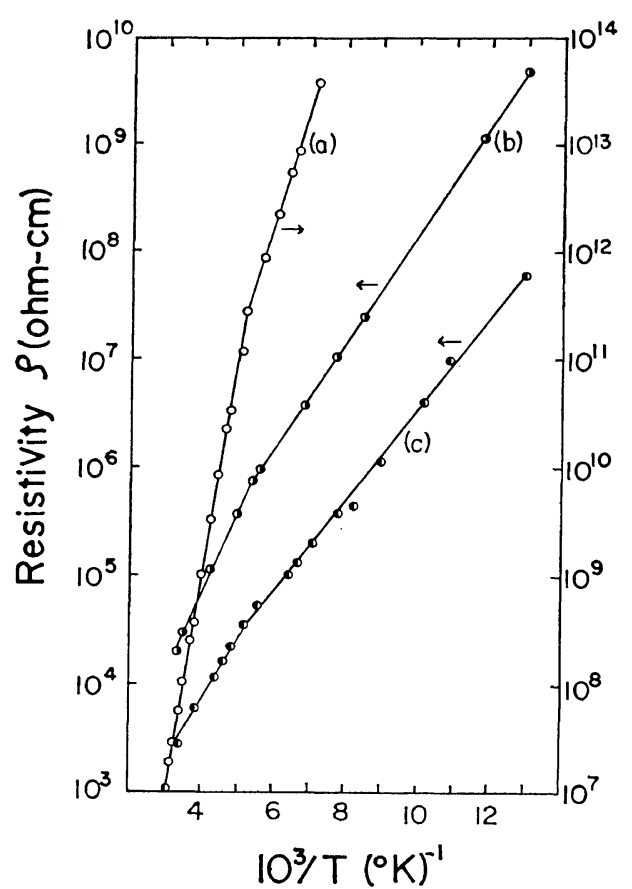

Figure 2. Plots of $\log$ resistivity $v s$. $10^{3} / T$ for samples II and III: (a), sample II; (b), sample $\mathrm{III}_{0.15}$; (c), sample III $_{0.40}$. Sample notations, see Figure 1.

In Figure 5, the relative dielectric constant of the polycation chloride (I) is plotted against frequency. As evidenced from the small dielectric constant of this material, the contribution of chloride anions to the dielectric constant is smaller than the contribution of the TCNQ anion radical and especially when compared with that of the anion radical complexed with the neutral TCNQ molecule. Also, as the elemental analysis shows almost no residual chlorine ions in the simple salt (II), we can exclude ionic

Table I. Specific resistivities and activation energies of simple and complex salts

\begin{tabular}{lccccc}
\hline Sample & Doping ratio & TCNQ content & b,$w t \%$ & $\rho, 25^{\circ} \mathrm{C}, \Omega \mathrm{cm}$ & \multicolumn{2}{c}{$E_{\mathrm{a}}, \mathrm{eV}^{\mathrm{c}}$} \\
\hline $\mathrm{II}$ & 0 & 60 & $2.5 \times 10^{7}$ & $0.41\left(60--83^{\circ} \mathrm{C}\right)$ & $0.22\left(-83--135^{\circ} \mathrm{C}\right)$ \\
III $_{0.15}$ & 0.15 & 63 & $2.1 \times 10^{4}$ & $0.15\left(21--88^{\circ} \mathrm{C}\right)$ & $0.10\left(-88--196^{\circ} \mathrm{C}\right)$ \\
III $_{0.40}$ & 0.40 & 67 & $6.0 \times 10^{3}$ & $0.11\left(21--83^{\circ} \mathrm{C}\right)$ & $0.083\left(-83--196^{\circ} \mathrm{C}\right)$ \\
\hline
\end{tabular}

a For sample notation, see Figure 1 .

b Densities of TCNQ crystal and Sample II are estimated to be approximately 1.35 and 1.20 , respectively, by observing their floating behavior in halogenated solvents. Consequently, the volume fraction of TCNQ molecules is slightly lower than the weight fraction.

c $\rho=\rho_{0} E_{\mathrm{a}} / k T$.

* Doping ratio means the molar ratio of neutral

TCNQ/TCNQ anion radical. 


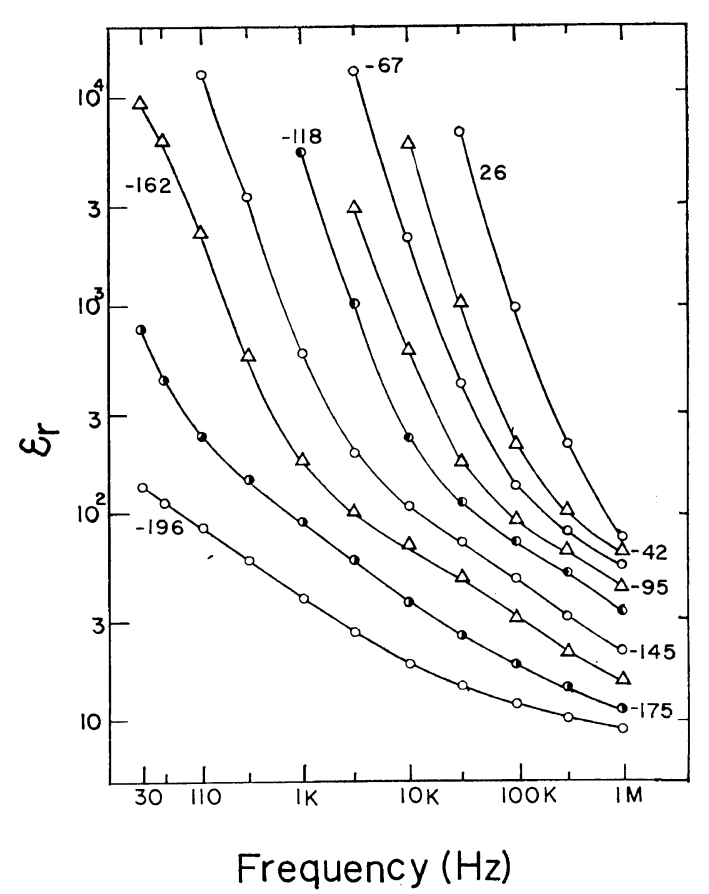

Figure 3. Plots of relative dielectric constant $\varepsilon_{\mathrm{r}} v s$. frequency for sample III $_{0.40}$ at various temperatures.

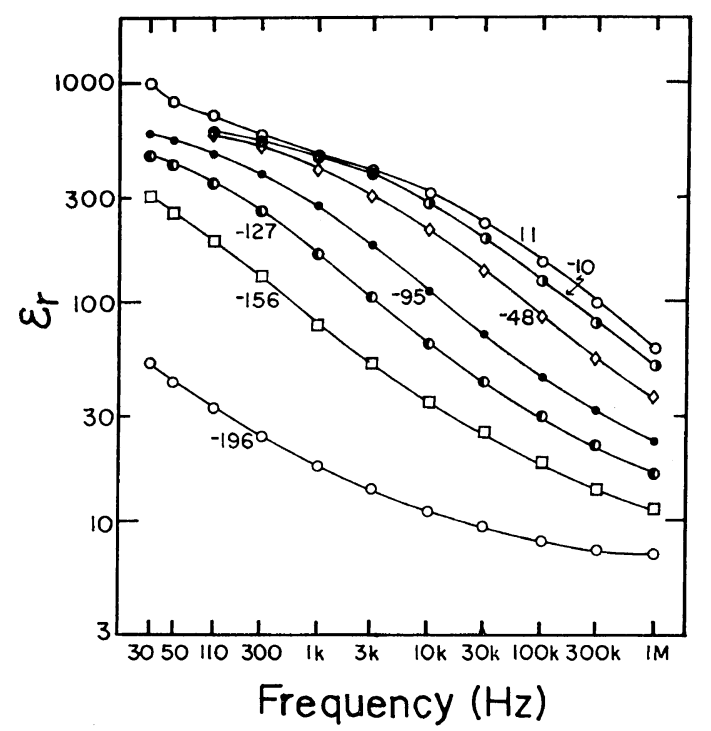

Figure 4. Plots of relative dielectric constant $\varepsilon_{\mathrm{r}} v s$. frequency for sample $\mathrm{III}_{0.15}$ at various temperatures.

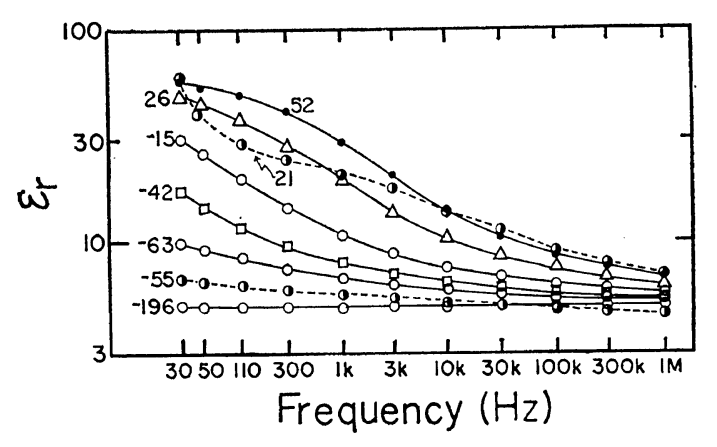

Figure 5. Plots of relative dielectric constant $\varepsilon_{\mathrm{r}} v s$. frequency for samples I and II at various temperatures: Dotted line, sample I; solid line, sample II.

Table II. Effect of field intensity on dielectric constant and conductance of complex salts

\begin{tabular}{rrrrr}
\hline Sample & $\begin{array}{c}\text { Temp, Frequen- } \\
{ }^{\circ} \mathrm{C}\end{array}$ & $\varepsilon_{4 \cdot 0 \mathrm{~V}} / \varepsilon_{0.35 \mathrm{~V}}{ }^{\mathrm{b}}$ & $G_{4.0 \mathrm{~V}} / G_{0.35 \mathrm{~V}}{ }^{\mathrm{C}}$ \\
\hline $\mathrm{III}_{0.15}$ & 11 & 50 & 0.89 & 1.084 \\
& 11 & 1000 & 0.96 & 1.036 \\
$\mathrm{III}_{0.40}$ & -43 & 10000 & 0.94 & 1.001 \\
& -67 & 3000 & 0.75 & 1.010 \\
\hline
\end{tabular}

a For sample notation, see Figure 1.

b Ratio of dielectric constants. Subscript means intensity of applied AC field.

c Ratio of $\mathrm{AC}$ conductances.

polarization due to chlorine ions in (II) and (III) polymers. Considering the low contribution of electronic polarization (2-3) and of orientation polarization (order of 10) to the dielectric constant of homogeneous organic materials which contain no ionic or electronic carriers, the high dielectric constants observed in our polymers are supposed to be induced by electronic carriers abundantly dispersed in the polymers.

The effect of the field intensity on the dielectric constant and conductance was observed in the complex salts. The results are shown in Table II. As the field intensity increases, the dielectric constant decreases moderately and the conductance increases slightly. Such a variation of dielectric constant with field is unusual, since the dielectric constant is independent over a wide range of field strength for most organic polymers.

The results exhibited above are quite similar 
to the dielectric properties reported by H.A. Pohl $^{2}$ for polyacene radical quinone polymers: i.e., (a) unusually high dielectric constant and (b) field, temperature, and frequency dependencies of the dielectric constant.

The dielectric loss tangent of (II) and (III) are shown in Figures 6-8. While a $\tan \delta$ maximum is observed for sample $\mathrm{III}_{0.40}$, it is not observed for samples II and $\mathrm{III}_{0.15}$. However, we can also observe a $\tan \delta$ maximum for samples II and $\mathrm{III}_{0.15}$ when the DC conductance is subtracted from the apparent AC conductance. When the same correction is applied for sample III $_{0.40}$, the relaxation frequencies shift slightly to higher frequencies. Plots of the logarithm of the relaxation frequency $f_{\mathrm{m}}$ against the reciprocal of absolute temperature are shown in Figure 9. The curves of the $\log f_{\mathrm{m}}$ vs. $1 / T$ are linear, and breaks are found at around the same temperature as the breaks of the resistivity plot of Figure 2. The activation energies $\Delta H$ for the polarization process, calculated by Eyring's equation,

$$
\ln f_{\mathrm{m}}=-\frac{\Delta H}{k T}+\mathrm{const}
$$

where $k$ is Boltzmann's constant, are shown

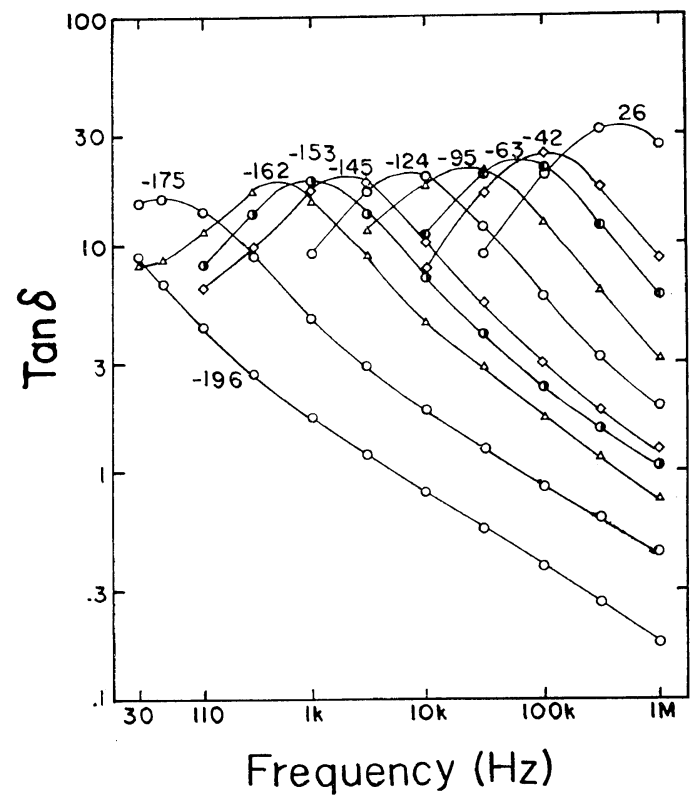

Figure 6. Dielectric loss tangent $\tan \delta v s$. frequency for sample $\mathrm{III}_{0.40}$ at various temperatures.

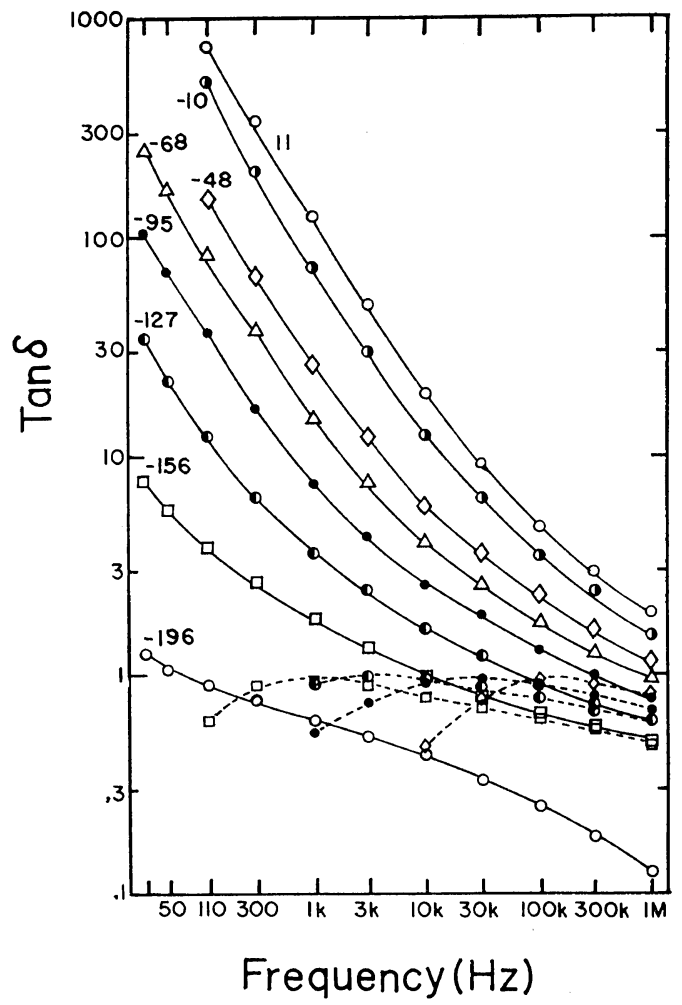

Figure 7. Dielectric loss tangent $\tan \delta v s$. frequency for sample $\mathrm{III}_{0.15}$ at various temperatures. Dotted lines show the corrected $\tan \delta$ value obtained by subtracting the DC conductance from the observed AC conductance. When the AC conductance becomes constant at low frequency, this conductance is taken as the DC conductance. Otherwise, the observed DC conductance is used for the correction.

in Table III. The activation energies of polarization agree well with those of electrical conduction (Table I), suggesting the same mechanistic origin for both phenomena. Thus, we consider mobile carriers to be responsible for the high dielectric constant of our polymers.

\section{Structural Model}

Electronic conduction in semiconducting polymer salts consisting of polymer cation and TCNQ anion radical doped with some amount of neutral TCNQ has usually been considered by following the model shown in Figure $10 .^{8}$ In principle, this means that TCNQ anion radicals hanging to the cationic sites of the polymer chain make a columnar stacking of TCNQ together with the added neutral TCNQ molecules 


\section{S. Ikeno, K. Matsumoto, M. Yokoyama, and H. Mikawa}

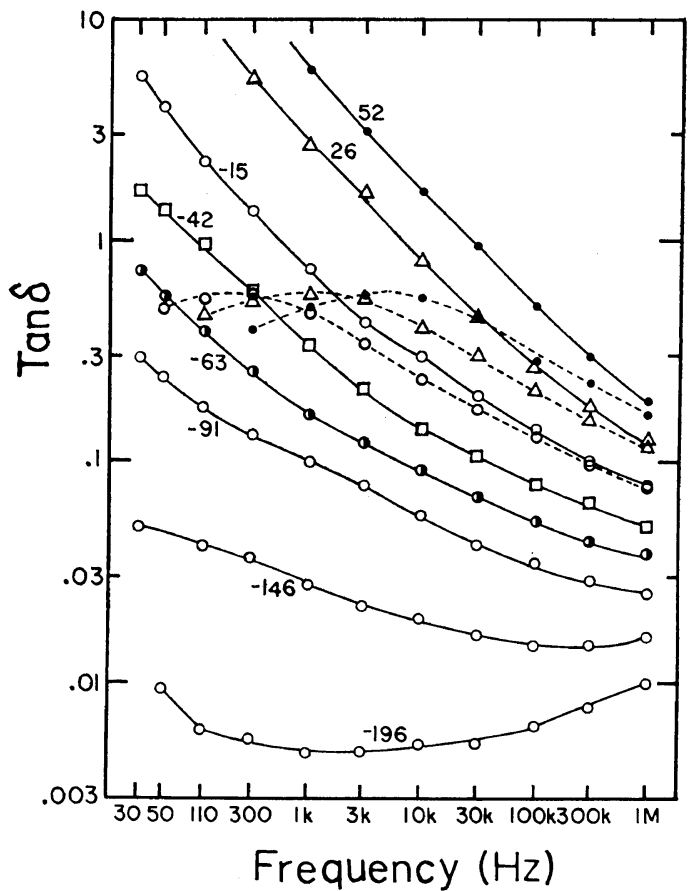

Figure 8. Dielectric loss tangent $\tan \delta$ vs. frequency for sample II at various temperatures. Dotted lines show the corrected $\tan \delta$ value obtained by subtracting the DC conductance from the observed $\mathrm{AC}$ conductance.

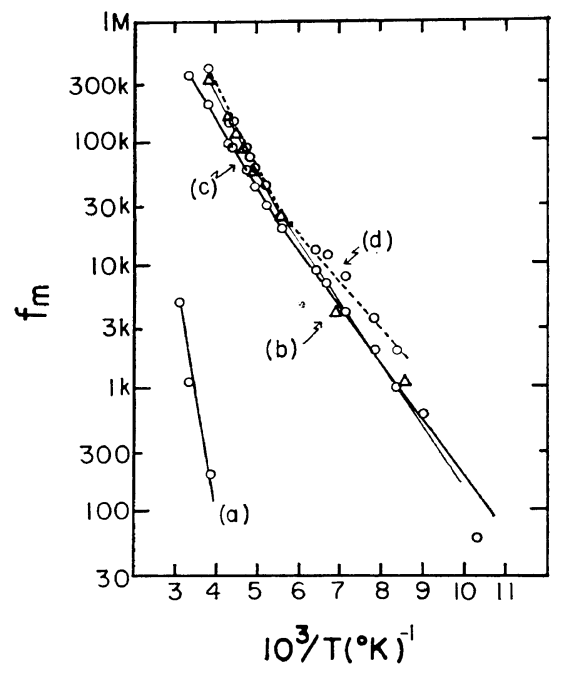

Figure 9. $\log f_{\mathrm{m}}$ vs. $10^{3} / T$ for samples $\mathrm{II}, \mathrm{III}_{0.15}$, and $\mathrm{III}_{0.40}$ : (a), sample II; (b), sample $\mathrm{III}_{0.15}$; (c), sample $\mathrm{III}_{0.40}$; (d), sample $\mathrm{III}_{0.40}$. The relaxation frequencies $f_{\mathrm{m}}$ in (c) and (d) are obtained from the plots of uncorrected and corrected $\tan \delta$ values, respectively.
Table III. Activation energies of polarization process

\begin{tabular}{lll}
\hline Sample & \multicolumn{2}{c}{$\Delta H,{ }^{\mathrm{a}} \mathrm{eV}$} \\
\hline II & $0.39\left(52--15^{\circ} \mathrm{C}\right)$ & \\
III $_{0.15}$ & $0.12\left(-10--95^{\circ} \mathrm{C}\right)$ & $0.10\left(-95--156^{\circ} \mathrm{C}\right)$ \\
III $_{0.40}$ & $0.13\left(-12--95^{\circ} \mathrm{C}\right)$ & $0.077\left(-95--162^{\circ} \mathrm{C}\right)$ \\
III $_{0.40^{\mathrm{b}}}$ & $0.11\left(-12--95^{\circ} \mathrm{C}\right)$ & $0.091\left(-95--162^{\circ} \mathrm{C}\right)$
\end{tabular}

a $\Delta H$ for polarization as calculated by eq 1 .

b Relaxation frequency is obtained from $\tan \delta$ values without correction for the contribution of the DC conductance.

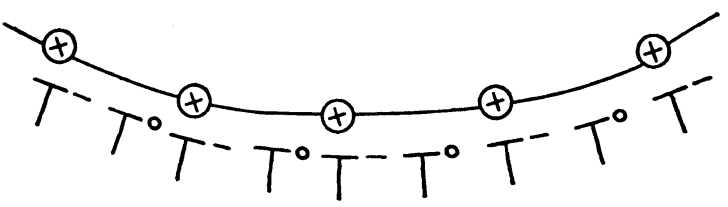

Figure 10. Structural model of polycation-TCNQ salt considered hitherto.

and this columnar stacking, although it has some irregularities, provides a pathway for electronic carriers. From the considerations which follow, this kind of model seems to be not so adequate, because TCNQ columnar stacking is over-emphasized in this model.

We have now a good accumulation of information about the crystal structures of ionic charge transfer salts and of anion radical salts of low molecular cations. ${ }^{12}$ These data show that a columnar structure requires rather severe conditions on the constituent ions. This is natural because the large coulombic repulsion between the anions in a column ${ }^{11}$ must be compensated by the polarization energy of the ambient molecules and by the coulombic stabilization due to the complicated cationic field. ${ }^{11}$ Thus, the delicate electrostatic energy balance leads in some cases to a so-called mixed stack structure. $^{13}$ Even in the case of a real columnar structure, the deformation of the columnar stacking to diadic to tetradic groupings of the anion radical molecules is quite common; ${ }^{14}$ there are a few exceptions, ${ }^{14}$ especially compounds which have monadic stacking with metallic conductivity.

In polycation polymers, the regularity of the cationic sites must be very low. In our polycationic salts of TCNQ, TCNQ molecules usually occupy up to $50 \%$ of the material by volume 
(Table I). The specific gravity of this material shows that the packing of TCNQ molecules and polymer cations is compact without leaving much free volume. Considering the high density of cations and the random distribution of such cations, the model schematically shown in Figure 11 in which TCNQ molecules are packed almost randomly in the polycation network seems more probable than the model shown in Figure 10. In the model shown in Figure 11, large TCNQ molecules locate themselves in suitable positions as determined by the complicated ambient integral electrostatic field, and it is impossible to identify the polycation polymer to which some specified TCNQ molecule belongs.

In TCNQ ionic crystals, the highest electrical

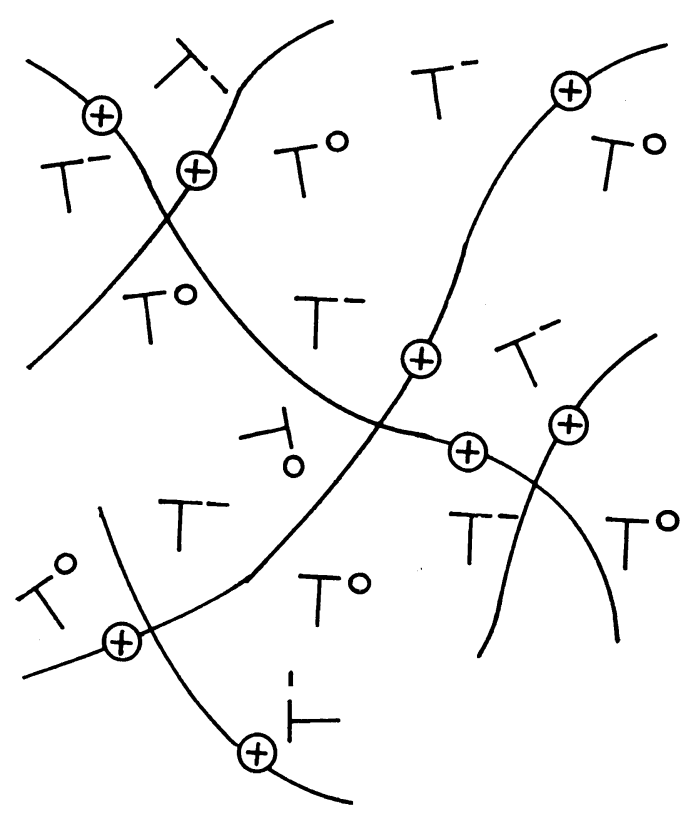

Figure 11. Structural model of polycation-TCNQ salt proposed in the present work-Large TCNQ molecules locate themselves in suitable positions as determined by the complicated ambient integral electrostatic field, and it is impossible to identify the polycation polymer to which some specified TCNQ molecule belongs. TCNQ anion radicals interact with $\mathrm{TCNQ}^{-} \mathrm{s}$ or with $\mathrm{TCNQ}^{\circ} \mathrm{s}$ in nearby positions and the electrons may delocalize in the TCNQ aggregates thus formed in forming a chargetransfer state, $\mathrm{TCNQ}^{\circ} \ldots \mathrm{TCNQ}^{=}$, or a chargeresonance state, $\mathrm{TCNQ}^{-} \cdot \ldots \mathrm{TCNQ}^{\circ} \leftrightarrow \mathrm{TCNQ}^{\circ} \ldots$ $\mathrm{TCNQ}^{-}$, transiently. conductivity is indeed in the direction of columnar stacking of TCNQ. However, some amount of conductivity, usually two orders smaller than the stacking direction, is observable to the other crystal directions. ${ }^{15}$ This means that some conduction is still possible along the direction of side-by-side stacking of TCNQ entities. There is thus no reason to assume in polymeric systems that electron migration occurs exclusively in the face-to-face stacking of TCNQ entities, as in Figure 10. In explaining the conductivity in TCNQ salts of cationic polymers, it seems that too much emphasis has been placed hitherto on the face-to-face stacking of TCNQ molecules and the electronic conduction through this stacking.

Thus, with respect to the electronic migration, each TCNQ molecule in Figure 11 may be said to be potentially "polyfunctional", in the meaning that-(1) from a TCNQ molecule, electronic migration may take place to limited numbers of other TCNQ molecules in so far as such molecules exist in nearby positions (Figure 12); (2) owing to the irregularities of the TCNQ arrangement in the material, one may be allowed to round up the difference of the ease of electronic migration and consider this equal; (3) on this simplification, every TCNQ molecule may be looked upon as having the same potential possibility of being connected electrically with several nearby TCNQ molecules. Figure 12 may explain this functionality.

In the model shown in Figure 11, electrical conduction from electrode to electrode may take place along the continuous chains of TCNQ molecules (continuous paths) in which TCNQ molecules are so arranged that through each neighboring TCNQ molecule at least some electronic migration is possible. In spite of the extremely low probability of the formation of such a long conductive chain, some overall conductivity may result owing to the enormous number of possible paths which start from one electrode and can reach the other. This type of reasoning is commonly used in explaining the conductivity of polymer blend systems of conductive particles or fibers. ${ }^{16}$ As will be discussed later and as is shown schematically by Figure 13, this will be understood if one applies to the material a quasi-lattice model in which the lattice volume is approximately equal 
S. Ikeno, K. Matsumoto, M. Yokoyama, and H. Mikawa

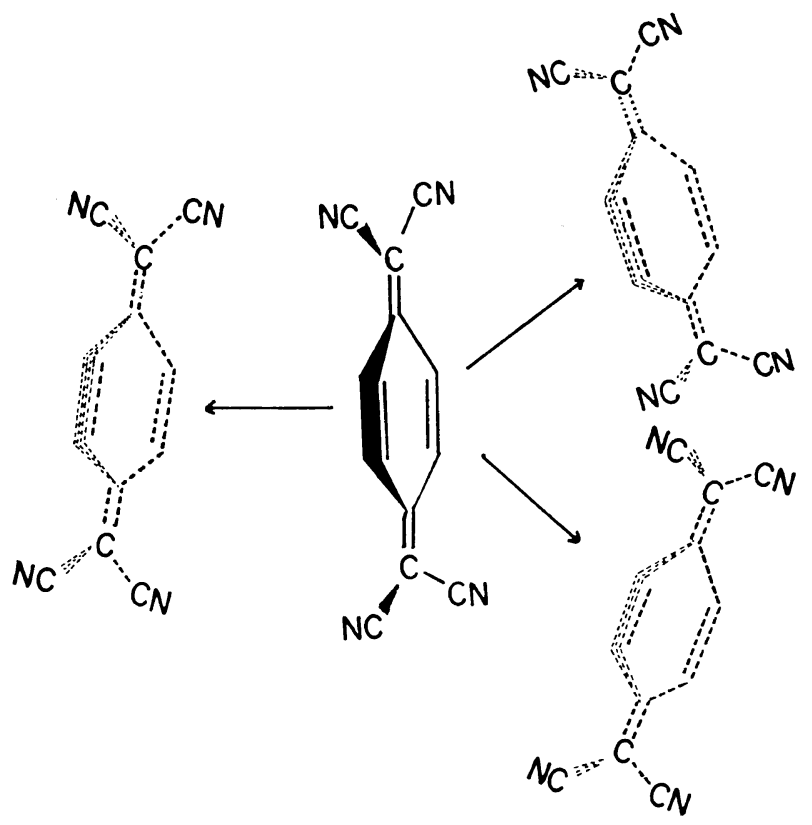

Figure 12. Schematic representation of "polyfunctionality" of TCNQ molecules. A TCNQ molecule has a limited number of TCNQ molecules to which electron transfer can take place in so far as molecules exist in nearby positions. In this meaning, TCNQ molecules could be considered to be potentially electrically "polyfunctional".

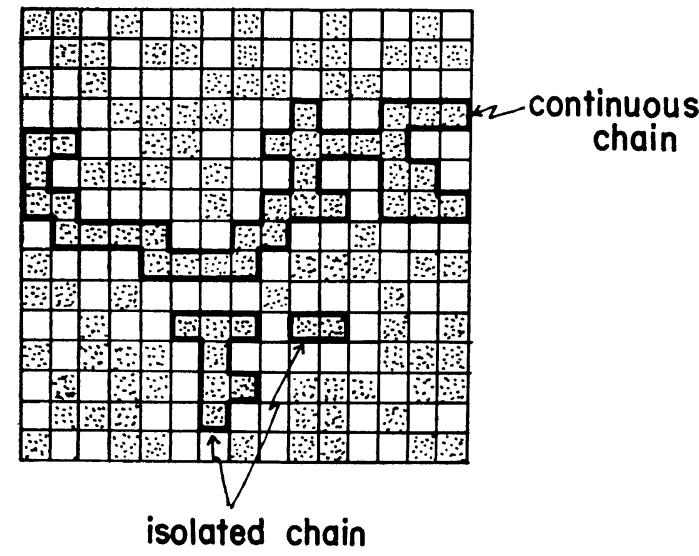

图=a $\square=b$

Figure 13. Two dimensional quasi-lattice model of the structure of polycation-TCNQ salt: a, lattice points occupied by TCNQ molecules; $b$, lattice points occupied by polycation chain.

to the volume of a TCNQ molecule and in which the probability of lattice occupation by a TCNQ molecule is equal to the volume fraction of
TCNQ molecules in the material. Obviously, it is not considered in this approximation that cationic sites are connected by polymer chains.

In this model, isolated chains of conductive TCNQ sequences (isolated paths) may also exist. Also, there may exist chains which start from one electrode but come to end at some place in between the electrodes, (branched paths). These chains can not contribute to the over-all DC conductivity of the material. When an electric field is applied, the isolated chains may, however, be polarized and contribute to the over-all dielectric polarization of the material. Thus, the material may be looked upon as a MaxwellWagner-type heterogeneous dielectric ${ }^{17}$ of molecular size.

In order to analyze the dielectric properties of the material with this mechanism, we must know the amount of "isolated" chains in the material. This is possible, although in very simplified form, as follows:-As is evident from Figures 12 and 13, every TCNQ molecule has the potential of forming a "conductive contact" with more than two neighboring TCNQ mo- 
lecules, and this probability of forming "conductive contact" may be assumed to be approximately equal to the volume fraction of TCNQ molecules in the material.

This calculation is possible by applying the mathematical treatment developed by P. J. Flory ${ }^{18}$ for molecular weight distribution and gelation in nonlinear polymers. According to his calculations, a monomer having $f$ reaction sites in a molecule ( $f$-functional monomer) can not have the possibility of growing into polymers of an infinite molecule (gel), in so far as the extent of reaction $\alpha$ does not exceed a critical value $\alpha_{\mathrm{c}}=1 /(f-1)$. Here, the extent of reaction $\alpha$ means the fraction of the reaction sites which enter into the reaction. Even after the extent of reaction has exceeded $\alpha_{\mathrm{c}}$, i.e., even after the beginning of gel formation, it can be shown theoretically that the probability of the formation of polymers of finite molecular size (sol) does still exist. Thus, for some value of $\alpha$, which exceeds $\alpha_{\mathrm{c}}$, the weight fraction of sol or finite molecules in the polymer is given by

$$
W_{\mathrm{s}}=\frac{(1-\alpha)^{2} \alpha^{\prime}}{\left(1-\alpha^{\prime}\right)^{2} \alpha}
$$

where $\alpha^{\prime}$ is the smallest root of the equation

$$
\beta=\alpha(1-\alpha)^{f-2} \text {. }
$$

For the given value of $\alpha$, eq 3 determines $\beta$; once $\beta$ is thus determined, $\alpha^{\prime}$ will be determined as the smallest $\alpha$ which satisfies eq 3. The weight fraction of gel can be obtained by the difference

$$
W_{\mathrm{g}}=1-W_{\mathrm{s}} .
$$

The weight fraction of $X$-mers is given by

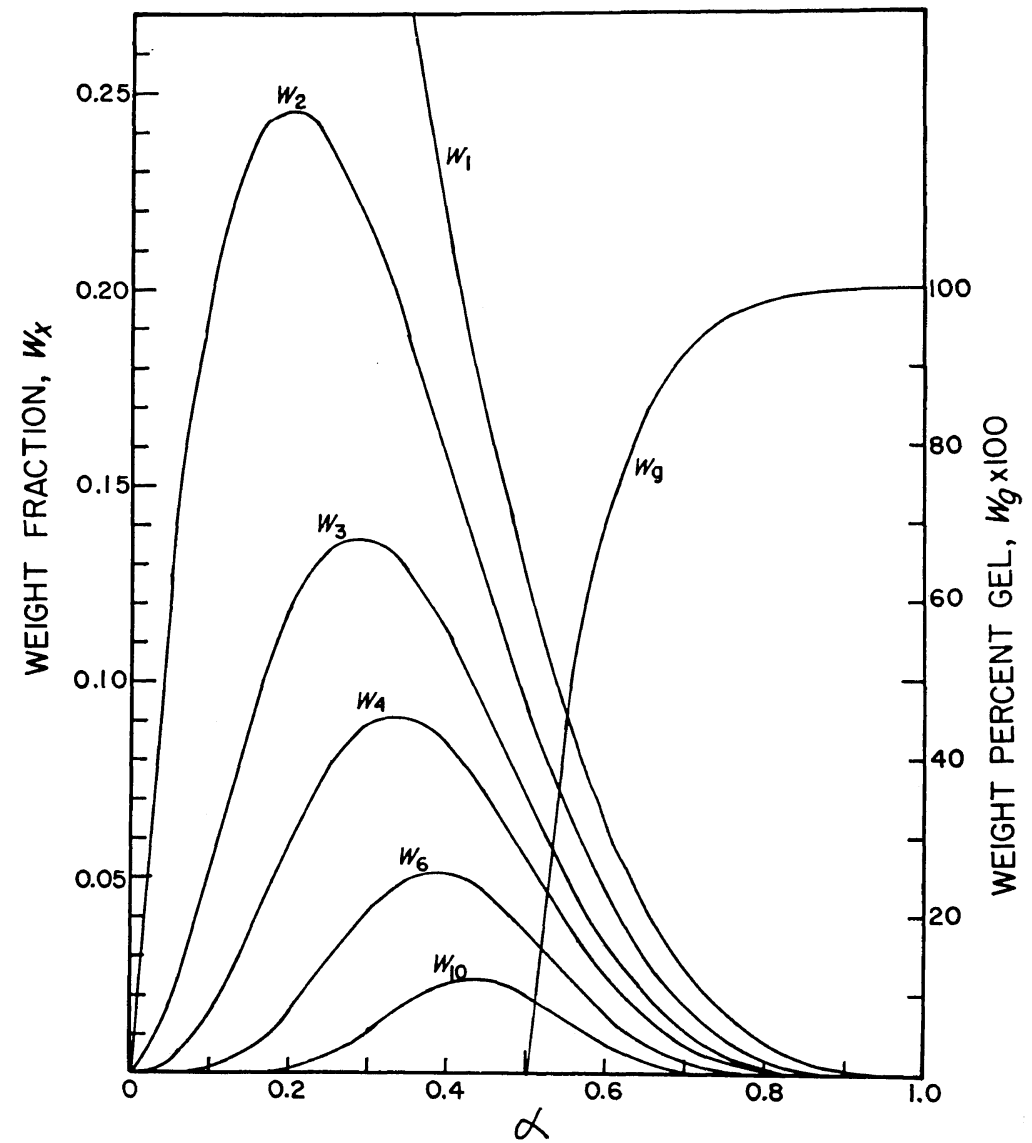

Figure 14. Weight fractions of various species in trifunctional condensation as.a function of extent of reaction $\alpha$ calculated by eq 4 and 5 . 


$$
W_{\mathrm{x}}=\frac{\left(1-\alpha^{\prime}\right)^{2}}{\alpha^{\prime}}\left[\frac{f(f x-x) !}{(x-1) !(f x-2 x+2) !}\right] \beta^{x} W_{\mathrm{s}}
$$

where $x$ is the number of monomer units in the molecule. The weight fraction of X-mers is plotted against $\alpha$ in Figure 14. As stated in the previous paragraph, if we replace the polyfunctional monomers and the extent of reaction $\alpha$ in Flory's theory by TCNQ molecules and the probability of forming "conductive contact" (i.e., the volume content of TCNQ molecule in the material), the isolated and continuous sequences of TCNQ molecules can be considered as sol and gel in Flory's theory, respectively. The monomer functionality $f$ corresponds to the functionality of TCNQ molecules stated already. Since the arrangements of TCNQ molecules are assumed to be random in our polycation-TCNQ salts, we expect that $f$ is larger than two, as illustrated in Figure 12. Considering the threedimensionality of coordination, we presume that the average functionality of TCNQ molecules is three or more. The extent of reaction, $\alpha$, means the probability of TCNQ molecules existing in nearby positions so that electron transfer is possible. Although it is hard to estimate this probability, we may be allowed to assume it to be equal to the volume content of TCNQ molecules in the material, because the occupation probability of nearby sites by TCNQ molecules becomes large with increasing amounts of TCNQ molecules. In this way, we may be able to make our model of polycation-TCNQ salts quantitative to some extent by applying Flory's gelation theory. ${ }^{19}$ If we assume $\alpha=0.60$ and $f=3$ for the simple salt, the weight content of various paths could be calculated, as shown in

Table IV. The weight fractions of various paths as calculated by the theory of gelation ${ }^{a}$

\begin{tabular}{|c|c|c|c|c|}
\hline 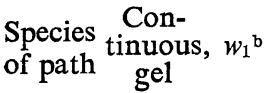 & $w_{2}$ & $w_{3}$ & $w$ & $w_{6}$ \\
\hline
\end{tabular}

$\begin{array}{llllllll}\text { Weight } & 0.704 & 0.064 & 0.046 & 0.033 & 0.025 & 0.015 & 0.007\end{array}$ fraction

a Values are calculated by eq 5 for $\alpha=0.60$ and $f=3$.

b $w_{x}$, the weight fraction of isolated paths. Subscript means the number of TCNQ molecules involved in a isolated path.
Table IV. The figures in the table seem reasonably able to explain the electrical results.

\section{Explanations of Experimental Results on the Basis of the Proposed Model}

Using the above model, we can qualitatively explain the observed conductive and dielectrical properties of our polymers in the following way.

The conductive and dielectrical properties of the polymers are considered to be determined by two main factors: i.e., 1) the fraction of continuous and isolated paths and 2) the nature of the conduction path. The fraction of various paths is discussed above. The nature of the conduction path means the degree of ease for mobile carrier generation and transportation in the conduction path. The electron transportation may take place by an activation process, as proposed by Tsubomura, ${ }^{9}$ et al., i.e., in simple salts, electrons move from one $\mathrm{TCNQ}^{-}$molecule to the adjacent $\mathrm{TCNQ}^{-}$molecules, forming a temporary charge-transfer state, $\mathrm{TCNQ}^{\circ} \cdot \mathrm{TCNQ}^{=}$, and in complex salts electrons move from one $\mathrm{TCNQ}^{-}$to adjacent neutral TCNQ molecules, forming a temporary charge-resonance state, $\mathrm{TCNQ}^{-} \ldots \mathrm{TCNQ}^{\circ} \leftrightarrow \mathrm{TCNQ}^{\circ} \cdot . \mathrm{TCNQ}^{-}$. In contrast to the conduction in simple salts, where Coulomb repulsive forces have to be overcome in forming the dianion state transiently, mobile carrier generation and transportation in complex salts must be easier. This is clearly shown in the experimental results, i.e., 1) a great increase of conductivity and dielectric constant and 2) a great decrease of activation energies for both electrical conduction and polarization, in the case where neutral TCNQ molecules are doped into simple salts. Thus the nature of the conduction path is found to be an important factor which affects the conductive and dielectrical properties of the polymers. Electron transfer in continuous paths leads to electrical conduction, and electron transfer in isolated paths or in branched paths with path ends in the bulk may contribute to polarization. Because of the kinetic and transitory nature of the activated electrons which transfer to adjacent TCNQ molecules, high temperature and low frequency are favorable for both electrical conduction and dielectric polarization. As the field strength is increased, the possibility of electron transfer 
may increase between TCNQ molecules, which had originally been considered impossible. Mathematically, this increase corresponds to the increase of the extent of reaction, $\alpha$. Consequently, the decrease of dielectric constant and the increase of AC conductance with field, shown in Table II, could be explained qualitatively by the decrease of isolated and branched paths and the increase of continuous paths.

As already pointed out, the dielectric properties of our polymers could be explained by a Maxwell-Wagner-type polarization of conductive particles of molecular scale dispersed in an insulating matrix. In our polymers, the isolated chains and branched chains of TCNQ molecules provide these conductive particles. Sillars ${ }^{20}$ has developed a theory of dielectrics for a heterogeneous system of suspension of ellipsoids of rotation and derived an expression for the relaxation time $\tau$. For the polarization along the long axis,

$$
\tau=\frac{(n-1) \varepsilon_{1}+\varepsilon_{2}}{4 \pi \sigma_{2}}
$$

where $\varepsilon_{1}$ and $\varepsilon_{2}$ are the dielectric constants of the insulating phase and the conducting particles, respectively, $\sigma_{2}$ is conductivity of the particles, and $n$ is a shape factor of the ellipsoid. Large $n$ means a long ellipsoid. The conductivity of the insulating material is ignored. Then the relaxation frequency $f_{\mathrm{m}}$ is given by

$$
f_{\mathrm{m}}=\frac{2 \sigma_{2}}{(n-1) \varepsilon_{1}+\varepsilon_{2}} .
$$

Since the change of $\varepsilon_{1}$ and $\varepsilon_{2}$ with temperature is considered negligible when compared with the change of $\sigma_{2}$, the change of $f_{\mathrm{m}}$ with temperature depends mainly on the change of $\sigma_{2}$. In Figure $15, \log f_{\mathrm{m}}$ is plotted against $\log \sigma$ at different temperatures for samples II, $\mathrm{III}_{0.15}$, and $\mathrm{III}_{0.40}$. A linear relationship is obtained in all plots, and the slopes of the respective lines are approximately 45 degrees. This result may support that the dielectric properties of the polymeric TCNQ salts could be qualitatively explained by Maxwell-Wagner polarization of the isolated and branched TCNQ chains in the polymer, although the volume fraction of conductive particles is large in our polymer system.

The specimens studied here were in a tablet form, prepared by pressing a fine powder of the polymers. There might exist, therefore, a possibility of another type of Maxwell-Wagner

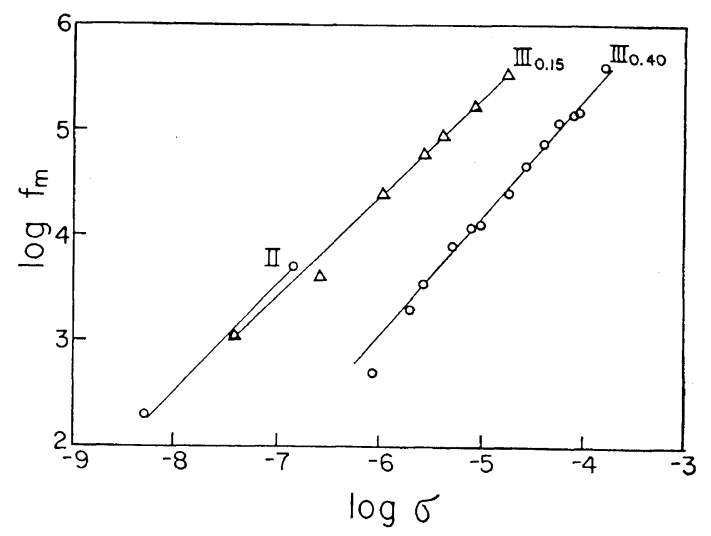

Figure 15. Plots of log relaxation frequency $\left(f_{\mathrm{m}}\right)$ vs. $\log \mathrm{DC}$ conductivity for samples $\mathrm{II}, \mathrm{III}_{0.15}$, and $\mathrm{III}_{0.40}$ at various temperatures.

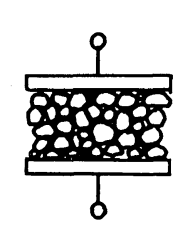

(a)

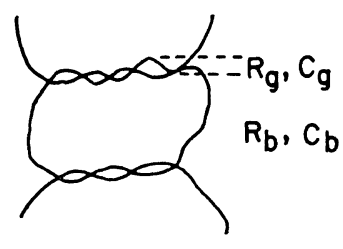

(b)

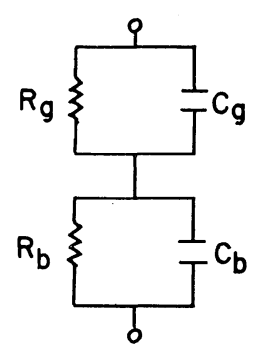

(c)

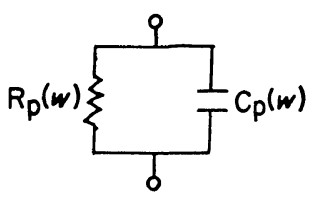

(d)

Figure 16A. Equivalent network system of powdered organic semiconductors: (a), representation of pressed powder sample between capacitor plates; (b), enlarged view of the void between grains. $R_{\mathrm{b}}$ and $C_{\mathrm{b}}$ show particle resistance and capacitance and $R_{\mathrm{g}}$ and $C_{\mathrm{g}}$ show gap resistance and capacitance, respectively; (c), (d), equivalent network systems. 

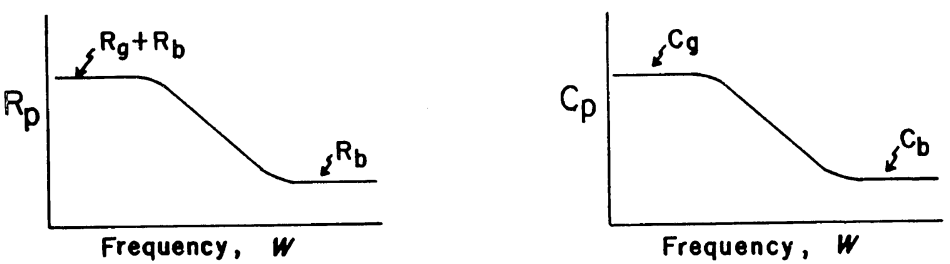

Figure 16B. Frequency dependence of effective parallel resistance and capacitance for the equivalent network system.

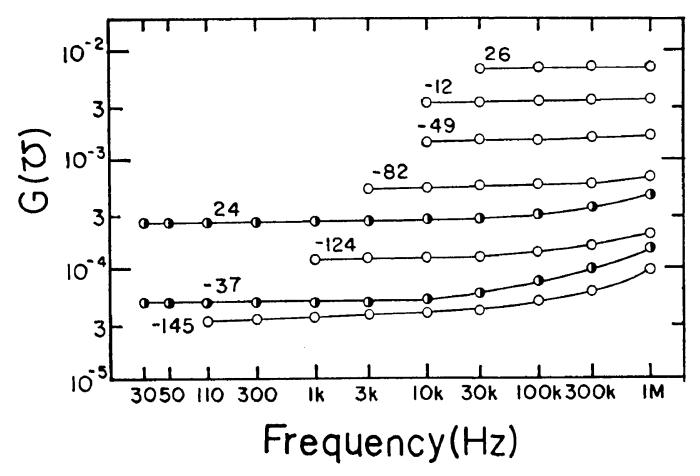

Figure 17. Plots of $\mathrm{AC}$ conductance vs. frequency for sample $\mathrm{III}_{0.15}(\mathrm{O})$ and sample $\mathrm{III}_{0.40}(\bigcirc)$ at various temperatures.

polarization which is observed sometimes in pressed powdered organic semiconductors: ${ }^{21}$ that due to grain boundaries. An equivalent network system $^{21}$ like Figure 16A has frequently been utilized in analyzing the electrical behavior in such systems. In such a type of MaxwellWagner polarization, both capacitance and resistance should in general exhibit a dispersive effect at or near the same frequency, as shown in Figure 16B. However, as shown in Figure 17 , the conductivity of our polymers does not show any dispersion in the frequency range where the dielectric constant shows a dispersion. Thus, in our material, dielectric dispersion due to grain boundaries could be ruled out.

In conclusion, the dielectric and conductive properties of polycation-TCNQ salts are reasonably explained by the proposed structural model. Electrical conductivity and dielectric constant depend on the number and nature of the continuous and isolated conductive sequences of TCNQ molecules. Assuming the isolated sequences to be conducting ellipsoids, the dielectric polarization could be explained by a microscale Maxwell-Wagner mechanism.
Acknowledgment. The authors wish to express their gratitude to Dr. S. Saito of the Electrotechnical Laboratory and Dr. S. Uemura of Osaka University for their valuable discussions. One of them (S.I.) is grateful to the Managing Director of Matsushita Electric Works Co. Ltd., K. Kobayashi, and Manager of Chemical Products Section, T. Murakami, for giving him the opportunity of doing this work at Osaka University.

\section{REFERENCES}

1. J.E. Katon, "Organic Semiconducting Polymers," Dekker, New York, N.Y., 1968.

2. Ya. M. Paushkin, T.P. Vishnyakova, A. F. Lunin, and S. A. Nizova, "Organic Polymeric Semiconductors," John Wiley and Sons, New York, N.Y., 1974.

3. R. Rosen and H. A. Pohl, J. Polym. Sci., Part $A-1,4,1135$ (1966).

4. R. D. Hartman and H. A. Pohl, ibid., Part A-1, 6, 1135 (1968).

5. J. R. Wyhof and H. A. Pohl, ibid., Part A-2, 8, 1741 (1970).

6. L. R. Melby, R. L. Harder, W. R. Hertler, W. Mahler, R. E. Benson, and W. E. Mochel, $J$. Am. Chem. Soc., 84, 3374 (1962).

7. J. H. Lupinski, K. D. Kopple, and L. J. Hertz, J. Polym. Sci., Part C, 16, 1561 (1967).

8. K. Mizoguchi, T. Suzuki, E. Tsuchida, and I. Shinohara, Nippon Kagaku Kaishi, 1973, 1760.

9. K. Nakatani, T. Sakata, and H. Tsubomura, Bull. Chem. Soc. Jpn., 48, 657 (1975).

10. K. Mizoguchi, T. Suzuki, E. Tsuchida, and I. Shinohara, Nippon Kagaku Kaishi, 1973, 1751.

11. A. G. Garito and A. J. Heeger, Account Chem. Res., 7, 232 (1974).

12. I. F. Shehegolov, Phys. Stat. Sol., 12, 9 (1972).

13. A. W. Hanson, Acta Cryst., 19, 610 (1965).

14. H. Kobayashi, T. Danno, and Y. Saito, ibid., B29, 2693 (1973).

15. W. J. Siemons, P. E. Bierstedt, and R. G. Kepler, J. Chem. Phys., 39, 3523 (1963). 
16. O. Maeda, G. Yamaki, and Y. Katayama, Kobunshi Ronbunshu, 32, No. 1, 42 (1975).

17. K. W. Wagner, Arch. f. Elektrotech., 2, 371, 374, 383 (1914).

18. P. J. Flory, "Principles of Polymer Chemistry," Cornell University Press, Ithaca, N.Y., 1953, Chapter 9.
19. For explaining electrical conductivity of carbon black filled polymers, Flory's gelation theory was used by Bueche. F. Bueche, J. Appl. Phys., 43, No. 11, 4837 (1972).

20. R. W. Sillars, J.I.E.E., 80, 378 (1937).

21. C. M. Huggins and A. H. Sharbaugh, J. Chem. Phys., 38, 393 (1963). 